\title{
The use of panel testing in familial breast and ovarian cancer
}

\author{
Authors: Matina Prapa, ${ }^{\mathrm{A}}$ Joyce Solomons ${ }^{\mathrm{B}}$ and Marc Tischkowitz ${ }^{\mathrm{A}, \mathrm{C}}$
}

\begin{abstract}
Advances in sequencing technology have led to the introduction of panel testing in hereditary breast and ovarian cancer. While direct-to-consumer testing services have become widely available, the clinical validity of many of the genes on panel tests remains contentious and risk management guidelines are often lacking. This article gives an overview of advantages with panel testing as well as important challenges, including clinical translation of test results.
\end{abstract}

\section{Introduction}

High-throughput sequencing of a number of genes (via targeted next-generation sequencing) at a declining cost is rapidly replacing sequential testing of single genes in both public and commercial service laboratories. The use of large multi-gene disease-targeted panels (see Table 1) is a cost-effective approach, also allowing incorporation of our improved understanding of the genetic architecture of disease. In this review, we focus on hereditary breast and ovarian cancer as a paradigm to illustrate the benefits and pitfalls of multi-gene panel testing in the clinic.

\section{Susceptibility genes and panel testing}

Germline mutations in the BRCA1 and BRCA2 genes remain the most important cause of familial forms of breast and ovarian cancer. ${ }^{1}$ Identification of a deleterious $B R C A 1 / 2$ variant within a family has profound implications in risk management, including enhanced surveillance, prophylactic surgery and chemoprevention options. ${ }^{2}$ However, in many families with a strong history of breast and/or ovarian cancer, BRCA1/2 testing does not identify a causative mutation.

Since the discovery of the BRCA1 and BRCA2 genes in the mid-1990s, numerous other susceptibility genes for breast or ovarian cancer have been identified (Table 2). Rare mutations in CDH1, PTEN, STK11 and TP53 genes, associated with hereditary diffuse gastric cancer, Cowden syndrome, Peutz-

Authors: ${ }^{A}$ specialist registrar in medical genetics, East Anglian Medical Genetics Service, Cambridge, UK; ${ }^{B}$ consultant in medical genetics, Oxford Centre for Genomic Medicine, Oxford University Hospitals NHS Foundation Trust; ${ }^{C}$ reader and honorary consultant physician in medical genetics, Academic Department of Medical Genetics, University of Cambridge, Cambridge, UK
Jeghers syndrome and Li Fraumeni syndrome, respectively, can confer a high risk of breast cancer. ${ }^{1,3}$ Besides these, intermediate breast cancer risk genes, such as ATM, CHEK2, and PALB2, have been identified. ${ }^{1,3}$ Large-scale genotyping studies have also led to the identification of numerous low risk variants, predominantly consisting of single nucleotide polymorphisms in non-coding sequences. ${ }^{4,5}$ Inherited mutations in other genes, such as RAD51C, RAD51D and BRIP1, and mismatch-repair (MMR) genes ( $M L H 1, M S H 2$, MSH6, PMS2, EPCAM) that cause Lynch syndrome when mutated, also influence the risk of ovarian cancer. ${ }^{6}$

These genetic discoveries have occurred alongside major advances in sequencing technology that has led to the introduction in the clinic of simultaneous testing of panels of selected multiple genes for hereditary breast and ovarian cancer predisposition. ${ }^{1}$ In theory, panel testing for hereditary breast and ovarian cancer allows for a more comprehensive

\section{Key points}

Additional susceptibility genes for familial breast and ovarian cancer have been identified since the discovery of the BRCA1/2 genes in the mid-1990s.

Deleterious mutations confer different levels of cancer risks, with lack of sufficient evidence for clinical actionability in many of these new genes.

Advances in sequencing technology led to the introduction of simultaneous multi-gene (panel) testing which is rapidly replacing sequential single-gene testing in public and commercial service laboratories.

Panel testing for familial breast/ovarian cancer has a slightly increased diagnostic yield compared to conventional BRCA1/2 testing.

There is a lack of consensus guidelines on panel testing, including eligibility criteria and clinical translation of results.

KEYWORDS: gene panel, breast cancer, ovarian cancer, genetic testing and hereditary cancer. 
Table 1. Examples of disease-targeted gene panels available via UKGTN where testing criteria and a gene dossier on prevalence and sensitivity of mutation detection is also included. ${ }^{10}$ Websites such as GeneReviews and OMIM (Online Mendelian Disorder in Man) provide clinicians with up-to-date information on genes associated with a Mendelian disorder in question. A comprehensive directory of available genetic testing services can then be accessed via webpages, such as UK Genetic Testing Network (UKGTN) and European Directory of DNA Diagnostic Laboratories (EDDNAL).

\begin{tabular}{|c|c|c|}
\hline Disease category & Disease & Genes \\
\hline \multirow[t]{2}{*}{ Hereditary cancer } & Familial breast/ovarian cancer & $\begin{array}{l}\text { ATM, BARD1, BRIP1, BRCA1, BRCA2, CDH1, CHEK2, PALB2, } \\
\text { PTEN, PIK3CA, STK11, TP53 }\end{array}$ \\
\hline & $\begin{array}{l}\text { Familial bowel cancer including hereditary } \\
\text { nonpolyposis colorectal cancer (HNPCC) } \\
\text { phenotype or polyposis }\end{array}$ & $\begin{array}{l}\text { APC, BMPR1A, POLD1, POLE, KIT, MLH1, MSH2, MSH6, } \\
\text { MUTYH, PDGFRA, PMS2, GALNT12, STK11, SMAD4 }\end{array}$ \\
\hline \multirow[t]{2}{*}{ Cardiac disorders } & Familial hypertrophic cardiomyopathies & $\begin{array}{l}\text { ACTC1, CSRP3, FHL1, GLA, LAMP2, MYBPC3, MYL2, MYL3, } \\
\text { MYLK2, MYH6, MYH7, NEXN, PLN, PRKAG2, SLC25A4, TTN }\end{array}$ \\
\hline & Aortopathy disorders & $\begin{array}{l}\text { ACTA2, COL3A1, FBN1, FBN2, MYH11, SMAD3, SLC2A10, } \\
\text { TGFB2, TGFB3, TGFBR1, TGFBR2 }\end{array}$ \\
\hline \multirow[t]{2}{*}{$\begin{array}{l}\text { Neurological and } \\
\text { neuromuscular disorders }\end{array}$} & Early infantile epileptic encephalopathy & $\begin{array}{l}\text { ALDH7A1, ARHGEF9, ARX, BTD, CHD2, CNTNAP2, CDKL5, } \\
\text { FOXG1, GABRB3, GABRG2, GLUD1, GRIN2B, HCN1, POLG }\end{array}$ \\
\hline & Congenital muscular dystrophies & $\begin{array}{l}\text { B3GALNT2, CHKB, COL4A1, COL4A2, COL6A1, COL6A2, } \\
\text { COL6A3, COL12A1, DOLK, DPM1, DPM2, DPM3, DAG1, } \\
\text { FKTN, FKRP, GMPPB, ITGA7, ITGA9, ISPD, LAMA2 }\end{array}$ \\
\hline
\end{tabular}

risk assessment, incorporating up-to-date knowledge on risk variants. Moreover, it may allow risk stratification in individuals who would not otherwise meet traditional testing criteria based on prior syndrome-focused approaches. However, panel testing is not without its limitations, including interpretation of results and subsequent risk management, especially in the absence of consensus guidelines.

\section{Availability and testing criteria}

Since the US Supreme Court decision in 2013 against Myriad Genetics' patent claims for BRCA1/2 testing, a number of gene panel testing services for hereditary breast and ovarian cancer, including BRCA1/2 genes have become available. ${ }^{1}$ These cover different combinations of genes with varying degrees of evidence of an association with breast and/or ovarian cancer which can be counterproductive where the evidence is limited. A case in point is BRIP1, which was first reported to be associated with breast cancer in $2006,{ }^{7}$ an observation that was not substantiated by a much larger study published ten years later. ${ }^{8}$ Yet this gene is still found on many commercially available hereditary breast cancer panels. ${ }^{1}$ In contrast, screening for single nucleotide polymorphisms is not typically included in panel tests, although it has been estimated that they can cumulatively confer a risk of $29 \%$ (by age 80 years for women in the top $1 \%$ for polygenic risk score ${ }^{4}$ ) which is comparable to moderate risk susceptibility genes (30\% and $28 \%$ for CHEK2 and $A T M$, respectively ${ }^{9}$ ).

While testing criteria for germline BRCA1/2 gene mutations are well defined, there are no equivalent guidelines for multigene panel testing in familial breast-ovarian cancer. In a UK-based survey conducted in 2016, 8 out of 19 participating centres offered panel testing in selected individuals following discussion at the weekly departmental meeting (personal communication by MT and JS). In some centres, BRCA1/2 testing was routinely run on a panel, with data on other genes reported upon the clinician's request. Eligibility criteria varied widely from centre to centre.

In the commercial setting, panel tests for breast cancer, such as the 17-gene BreastNext test by Ambry Genetics, are generally marketed to women who had uninformative BRCA1/2 testing and either have a personal history of early onset breast cancer (diagnosed $\leq 45$ years) or multiple close family members with breast and other cancers. Similarly, a 13-gene familial breast-ovarian cancer panel is available on the UKGTN with minimum requirements including the equivalent criteria for BRCA1/2 testing. ${ }^{10}$ These observations highlight the current inconsistencies in panel testing for breast and/or ovarian cancer susceptibility, in both clinical and commercial settings, and that access is largely dependent on availability and local guidelines.

\section{Diagnostic yield and variant interpretation}

Numerous studies have assessed the performance of targeted next-generation sequencing for the diagnosis of familial breast and ovarian cancer (Table 3 ). The analytical validity of panel testing was comparable to traditional sequencing methods (separate Sanger sequencing and deletion/duplication analyses of each gene). ${ }^{11,12}$ Overall, 'high-risk' breast cancer susceptibility genes are thought to account for $20-25 \%$ of familial breast cancer, with 'intermediate' risk genes explaining a further $5 \%$ and 'low risk' gene variants cumulatively covering an additional $14 \%$ of the familial risk. ${ }^{12}$

In a recent study by Desmond et al, ${ }^{13}$ panel testing in 1046 BRCA1/2-negative cases referred for hereditary breast-ovarian cancer predisposition led to identification of deleterious mutations in other hereditary cancer predisposition genes 
Table 2. Breast and ovarian cancer susceptibility genes (excluding BRCA1/2 genes).

Gene OMIM Associated syndrome and/or other
gene ID cancers

Breast cancer susceptibility genes

High risk

$\begin{array}{rrl}\text { CDH1 } & 602118 & \begin{array}{l}\text { Diffuse gastric cancer } \\ \text { Cowden syndrome (thyroid, endometrial } \\ \text { cancer) }\end{array} \\ \text { STK11 } & 601728 & \begin{array}{l}\text { Peutz-Jeghers syndrome (colon, pancreas, } \\ \text { ovarian sex cord-stromal tumours) }\end{array} \\ \text { TP53 } & 191170 & \begin{array}{l}\text { Li Fraumeni syndrome (sarcoma, } \\ \text { adrenocortical carcinoma, brain } \\ \text { tumours) }\end{array}\end{array}$

Intermediate risk

\begin{tabular}{|c|c|c|}
\hline ATM & 607585 & Ataxia telangiectasia (pancreas) \\
\hline CHEK2 & 604373 & \\
\hline NF1 & 613113 & $\begin{array}{l}\text { Neurofibromatosis Type I (malignant } \\
\text { tumours of peripheral nerve sheath, brain, } \\
\text { central nervous system) }\end{array}$ \\
\hline PALB2 & 610355 & Pancreas \\
\hline \multicolumn{3}{|c|}{ Unknown/disputed risk } \\
\hline BRIP1 & 605882 & Ovary (moderate risk) \\
\hline NBN & 602667 & Unknown \\
\hline RAD51C & 602774 & Ovary (moderate risk) \\
\hline RAD51D & 602954 & Ovary (moderate risk) \\
\hline \multicolumn{3}{|c|}{ Ovarian cancer susceptibility genes } \\
\hline BRIP1 & 605882 & \\
\hline EPCAM & 185535 & $\begin{array}{l}\text { Lynch syndrome (colon, endometrium, } \\
\text { stomach, small intestine, hepatobiliary } \\
\text { tract, urinary tract, brain, skin) }\end{array}$ \\
\hline MLH1 & 120436 & Lynch syndrome \\
\hline MSH2 & 609309 & Lynch syndrome \\
\hline MSH6 & 600678 & Lynch syndrome \\
\hline RAD51C & 602774 & \\
\hline RAD51D & 602954 & \\
\hline
\end{tabular}

in $3.8 \%$ of individuals, which was consistent with previous similar studies. ${ }^{12,14-16}$ In the majority of these (26/40), the identified variant was in 'low-moderate risk' breast-ovarian cancer susceptibility genes, such as CHEK2, with another eight cases harbouring mutations in genes associated with Lynch syndrome (which are not proven to cause breast cancer), and only three variants detected in high-risk breast cancer genes (all in CDH1). Apart from the increased likelihood of identifying a disease-causing mutation, panel testing can also lead to unexpected (incidental) findings in 'atypical' cases. For example, panel testing in 360 women with primary ovarian, peritoneal, or fallopian tube carcinoma led to identification of two MSH6 deleterious germline mutations in individuals with no family history of Lynch syndrome and three TP53 mutation carriers without a family history of Li-Fraumeni syndrome. ${ }^{16}$ Identifying such unexpected deleterious germline mutations could have implications for unaffected family members by way of cascading genetic tests, risk-assessment and future screening advice.

An important challenge for laboratories performing nextgeneration sequencing is the interpretation of identified variants, especially in genes other than $B R C A 1 / 2$ where available evidence is less well established. Although there is evidence of pathogenicity for protein-truncating variants (assumed to result in loss of function) for most genes included in hereditary breast-ovarian gene panels, the interpretation of missense variants is much more challenging. Missense variants in the coding region of genes that do not interfere with the reading frame are common and sometimes misinterpreted as pathogenic. Interpretation of these generally relies on use of in silico prediction tools, conservation data to assess if the variant is present in other species, familial segregation studies, and possibly tumour studies. ${ }^{1}$ Another useful tool can be the assessment of a variant frequency in affected and non-affected cohorts from multi-ethnic groups but this is dependent on the establishment and maintenance of international databases.

As a result of the above, the likelihood of identifying socalled variants of uncertain significance (VUS) is high. In a study by Kurian et al assessing the performance of panel testing in 175 women meeting BRCA1/2 gene testing criteria, sequencing of 39 genes led to identification of 2.1 VUS on average per participant, out of which the vast majority ( $88.8 \%)$ were novel. ${ }^{17}$ Similarly, one third (168/488) of women with breast cancer undergoing sequencing of 25 breast/ovarian cancer susceptibility genes harboured VUS, with as many as three variants found per patient. ${ }^{18}$ Most VUS will likely turn out to be non-pathogenic, but until this is known they will remain a source of considerable anxiety for the patient and their family.

\section{Clinical translation of results}

Most familial breast-ovarian cancer panels include moderate risk susceptibility genes for which consensus guidelines for risk management are not widely established and only beginning to be addressed in part. ${ }^{19,20}$ For many of these genes, there is limited evidence on the degree of associated cancer risk. For example, most existing data on CHEK2 gene derive from the 1100delC variant, which is the commonest truncating variant in northern European populations. ${ }^{1}$ Mutations in three other genes, BRIP1, RAD51C, and RAD51D, have been clearly associated with ovarian cancer but there is conflicting and limited evidence regarding their association with breast cancer. ${ }^{1}$

Thus a mutation-positive result arising from panel testing may not necessarily be clinically actionable. In the aforementioned study by Desmond et al, the most common deleterious mutations in BRCA1/2-negative cases were identified in low to moderate breast cancer risk genes with a recommended management change in the minority of these by either increased screening or prophylactic breast surgery (in the context of significant family history) based on National Comprehensive Cancer Network (NCCN) guidelines. ${ }^{13}$ Notably, there are no equivalent gene-specific NICE guidelines on the management 
Table 3. Summary of panel testing studies in familial breast and/or ovarian cancer.

\begin{tabular}{|c|c|c|c|c|c|}
\hline Study & Diseases & N subjects & $\begin{array}{l}\mathrm{N} \\
\text { genes }\end{array}$ & $\begin{array}{l}\mathrm{N} \text { deleterious } \\
\text { BRCA1/2 } \\
\text { mutations (\%) }\end{array}$ & $\begin{array}{l}\mathrm{N} \text { deleterious } \\
\text { mutations in all } \\
\text { other genes (\%) }\end{array}$ \\
\hline Walsh et al, $2011^{16}$ & Ovarian, fallopian tube, peritoneum & 360 & 21 & $63(17.5)$ & $22(6.1)$ \\
\hline Castera et al, $2014^{12}$ & Hereditary breast/ovarian cancer & 708 & 27 & $66(9.3)$ & $44(6.2)$ \\
\hline Kurian et al, $2014^{17}$ & Hereditary breast/ovarian cancer & 198 & 42 & $57(28.8)$ & $16(8.0)$ \\
\hline LaDuca et al, $2014^{14}$ & Ambry-tested, non BRCA1/2 & $\begin{array}{l}874 \text { (breast panel) } \\
223 \text { (ovarian panel) }\end{array}$ & $14-22$ & 0 (by definition) & $\begin{array}{l}65 \text { (7.4) for breast } \\
\text { panel } 16(7.2) \text { for } \\
\text { ovarian panel }\end{array}$ \\
\hline Couch et al, $2014^{22}$ & Triple-negative breast cancer & 1824 & $17 / 122$ & $204(11.1)$ & $67(3.7)$ \\
\hline Maxwell et al, $2014^{15}$ & Breast cancer $<40$ years, non $B R C A 1 / 2$ & 258 & 22 & 0 (by definition) & $31(11)$ \\
\hline Tung et al, $2015^{23}$ & Myriad tested & 1781 & 25 & $165(9.3)$ & $79(4.4)$ \\
\hline $\begin{array}{l}\text { Desmond et al, } \\
2015^{13}\end{array}$ & $\begin{array}{l}\text { Hereditary breast/ovarian cancer } \\
\text { (Invitae or Myriad tested), non } B R C A 1 / 2\end{array}$ & 1046 & $25-29$ & 0 (by definition) & $40(3.8)$ \\
\hline
\end{tabular}

of women who carry deleterious mutations in genes other than $B R C A 1 / 2$ and genes associated with rare conditions that carry a high risk of breast cancer (see Table 2$).^{21}$

Another important aspect of panel testing is the value of a negative result in an unaffected relative for a familial deleterious variant in an intermediate cancer risk gene. Incorporation of truncating mutations in three intermediate breast cancer risk genes (ATM, CHEK2, and PALB2) to the BOADICEA risk-prediction algorithm, illustrated that the reduction in risk for women whose mother carried a PALB2 mutation was comparable to a BRCA2 mutation, whereas negative testing for an ATM or CHEK2 familial mutation in the same scenario only led to a slight decrease in risk, even with a strong family history (Fig 1). ${ }^{9}$ In line with the above, a counselling framework has been recently proposed for moderate penetrance cancer-susceptibility mutations, suggesting that relatives who test negative for the familial mutation should be managed on the basis of their family history and may still warrant some enhanced surveillance. ${ }^{20}$
However, one could argue that if a woman's mutation status for these genes does not change her clinical management, there is little to be gained for testing them in the first place.

\section{Conclusion}

In the era of next-generation sequencing with continuously falling prices, the need for complex eligibility criteria for panel testing may be eliminated. Revision of the NICE guidelines in 2013 lowered the threshold for BRCA1/2 germline mutation testing, which is now offered to all individuals and their relatives who have a $>10 \%$ (instead of $20 \%$ ) likelihood of having a mutation based on risk algorithms, such as BOADICEA and the Manchester scoring system. ${ }^{21}$ Panel tests for familial breast/ ovarian cancer in the US typically cost around \$1500-3000 and include 10-30 genes, although the price is coming down rapidly with some companies offering panel tests for as little as $\$ 300$; in the $\mathrm{UK}, 13$-gene panel testing costs around $\mathfrak{E} 600-800$ versus

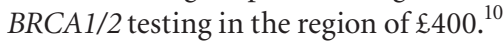

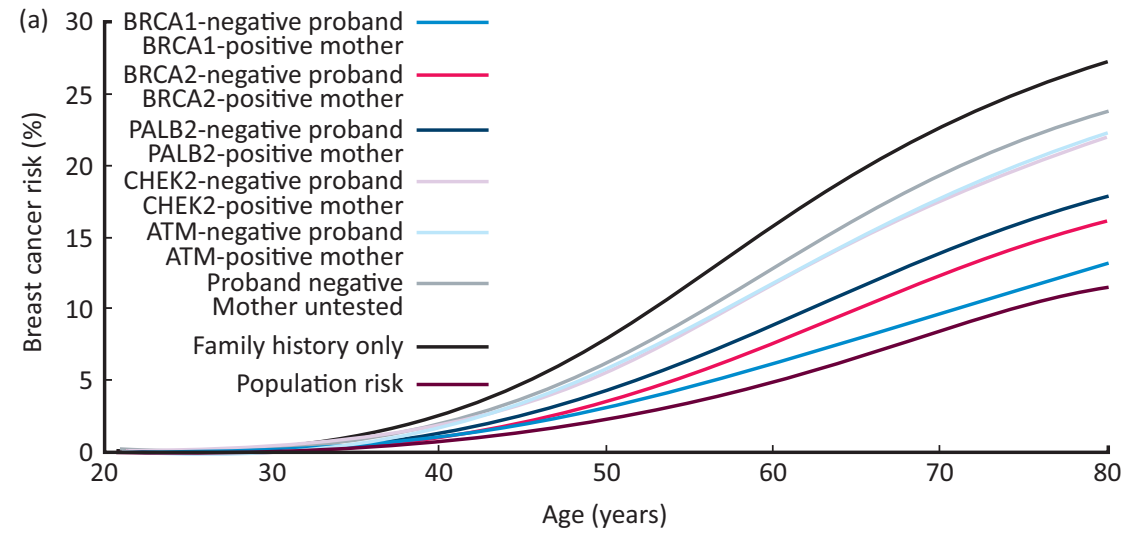

(b)

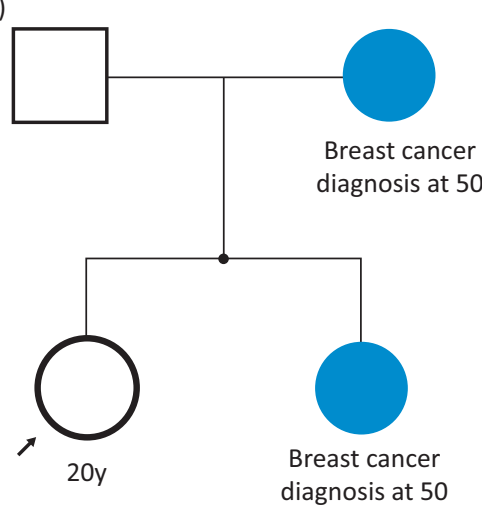

Fig 1. Value of negative testing for familial deleterious variants in moderate/high risk breast cancer genes. (a) shows the BOADICEA predicted breast cancer risk for negative testing (coloured curves) and based on family history alone (black curve). The proband, highlighted with an arrow in pedigree (b), is assumed to be a 20 year old woman in the United Kingdom. As illustrated, the reduction in risk following negative testing for a PALB2 familial mutation is comparable to a BRCA2 mutation whereas negative testing for an ATM or CHEK2 familial mutation is associated with a smaller decrease in risk. Image adjusted from Lee et al., with permission. ${ }^{9}$ 
Despite the above, panel testing is unlikely to overtake clinical acumen in the medium term, considering its limitations and our current gap in knowledge. The complexity of human variation, such as missense variants and incomplete penetrance due to environmental and other gene modifier factors, means that genetic data can at most add predictive value and always need to be interpreted within a clinical context. In the future, clinical geneticists may devolve diagnostic panel testing to mainstream specialties but would support them through interpretation of results and cascading genetic tests to relatives. Importantly, the absence of a pathogenic variant does not necessarily exclude the presence of familial and/or other inherited susceptibility factors and therefore, genetic counselling should still be considered an integral part of the process.

\section{References}

1 Easton DF, Pharoah PD, Antoniou AC et al. Gene-panel sequencing and the prediction of breast-cancer risk. N Engl J Med 2015;372:2243-57.

2 Petrucelli N, Daly MB, Feldman GL. Hereditary breast and ovarian cancer due to mutations in BRCA1 and BRCA2. Genet Med 2010;12:245-59.

3 Apostolou P, Fostira F. Hereditary breast cancer: the era of new susceptibility genes. Biomed Res Int 2013;2013:747318.

4 Mavaddat N, Pharoah PD, Michailidou K et al. Prediction of breast cancer risk based on profiling with common genetic variants. J Natl Cancer Inst 2015;107:djv036.

5 Michailidou K, Lindström S, Dennis J et al. Association analysis identifies 65 new breast cancer risk loci. Nature 2017, in press (doi: 10.1038/nature24284).

6 Lynch HT, Snyder C, Casey MJ. Hereditary ovarian and breast cancer: what have we learned? Ann Oncol 2013;24(Suppl 8):viii83-viii95.

7 Seal S, Thompson D, Renwick A et al. Truncating mutations in the Fanconi anemia J gene BRIP1 are low-penetrance breast cancer susceptibility alleles. Nat Genet 2006;38:1239-41.

8 Easton DF, Lesueur F, Decker B et al. No evidence that protein truncating variants in BRIP1 are associated with breast cancer risk: implications for gene panel testing. J Med Genet 2016;53:298-309.

9 Lee AJ, Cunningham AP, Tischkowitz $\mathrm{M}$ et al. Incorporating truncating variants in PALB2, CHEK2, and ATM into the BOADICEA breast cancer risk model. Genet Med 2016;18:1190-8.

10 UK Genetic Testing Network. https://ukgtn.nhs.uk/.

11 Lincoln SE, Kobayashi Y, Anderson MJ et al. A systematic comparison of traditional and multigene panel testing for hereditary breast and ovarian cancer genes in more than 1000 patients. J Mol Diagn 2015;17:533-44.
12 Castera L, Krieger S, Rousselin A et al. Next-generation sequencing for the diagnosis of hereditary breast and ovarian cancer using genomic capture targeting multiple candidate genes. Eur J Hum Genet 2014;22:1305-13.

13 Desmond A, Kurian AW, Gabree M et al. Clinical actionability of multigene panel testing for hereditary breast and ovarian cancer risk assessment. JAMA Oncol 2015;1:943-51.

14 LaDuca H, Stuenkel AJ, Dolinsky JS et al. Utilization of multigene panels in hereditary cancer predisposition testing: analysis of more than 2,000 patients. Genet Med 2014;16:830-7.

15 Maxwell KN, Wubbenhorst B, D’Andrea K et al. Prevalence of mutations in a panel of breast cancer susceptibility genes in BRCA1/2-negative patients with early-onset breast cancer. Genet Med 2015;17:630-8.

16 Walsh T, Casadei S, Lee MK et al. Mutations in 12 genes for inherited ovarian, fallopian tube, and peritoneal carcinoma identified by massively parallel sequencing. Proc Natl Acad Sci USA. 2011;108:18032-7.

17 Kurian AW, Hare EE, Mills MA et al. Clinical evaluation of a multiple-gene sequencing panel for hereditary cancer risk assessment. J Clin Oncol. 2014;32:2001-9.

18 Tung N, Lin NU, Kidd J et al. Frequency of germline mutations in 25 cancer susceptibility genes in a sequential series of patients with breast cancer. J Clin Oncol 2016;34:1460-8.

19 Daly MB, Pilarski R, Axilbund JE et al. Genetic/familial high-risk assessment: breast and ovarian, version 2.2015. J Natl Compr Canc Netw 2016;14:153-62.

20 Tung N, Domchek SM, Stadler Z et al. Counselling framework for moderate-penetrance cancer-susceptibility mutations. Nat Rev Clin Oncol 2016;13:581-8.

21 National Institute for Health and Care Excellence. Familial breast cancer: classification, care and managing breast cancer and related risks in people with a family history of breast cancer. Clinical guideline [cg164]. London: NICE, 2013. Available online at www.nice.org.uk/guidance/cg164.

22 Couch FJ, Hart SN, Sharma P et al. Inherited mutations in 17 breast cancer susceptibility genes among a large triple-negative breast cancer cohort unselected for family history of breast cancer. J Clin Oncol 2015;33:304-11.

23 Tung N, Battelli C, Allen B et al. Frequency of mutations in individuals with breast cancer referred for BRCA1 and BRCA2 testing using nextgeneration sequencing with a 25-gene panel. Cancer 2015;121:25-33.

Address for correspondence: Dr M Prapa, East Anglian Medical Genetics Service, Clinical Genetics, Box 134, Addenbrooke's

Treatment Centre, Level 6, Cambridge University Hospitals NHS Foundation Trust, Cambridge CB2 0QQ UK. Email: stamatia.prapa@addenbrookes.nhs.uk 